OPEN ACCESS

Edited by:

Alessandro Gozzetti,

University of Siena, Italy

Reviewed by:

Hiroyuki Takamatsu,

Kanazawa University, Japan

Stefania Oliva,

Ospedale Molinette, Italy

*Correspondence: Chor Sang Chim jcschim@hku.hk

Specialty section

This article was submitted to Hematologic Malignancies,

a section of the journal

Frontiers in Oncology

Received: 23 December 2018 Accepted: 13 May 2019

Published: 06 June 2019

Citation:

Yao Q, Bai Y, Orfao A and Chim CS (2019) Standardized Minimal Residual Disease Detection by Next-Generation

Sequencing in Multiple Myeloma.

Front. Oncol. 9:449.

doi: 10.3389/fonc.2019.00449

\section{Standardized Minimal Residual Disease Detection by Next-Generation Sequencing in Multiple Myeloma}

\author{
Qiumei Yao ${ }^{1}$, Yinlei Bai ${ }^{2}$, Alberto Orfao ${ }^{3}$ and Chor Sang Chim ${ }^{1 *}$ \\ ${ }^{1}$ Department of Medicine, Queen Mary Hospital, The University of Hong Kong, Pokfulam, Hong Kong, ${ }^{2}$ Institute for \\ Immunology and School of Medicine, Tsinghua University, Beijing, China, ${ }^{3}$ Department of Medicine and Cytometry Service \\ (Nucleus), Cancer Research Centre (IBMCC, USAL-CSIC) and CIBERONC, Institute for Biomedical Research of Salamanca \\ (IBSAL), University of Salamanca (USAL), Salamanca, Spain
}

Next-generation sequencing (NGS) has been applied to monitor minimal residual disease (MRD) in multiple myeloma (MM). Standardized DNA input and sequencing depth is essential for achieving a uniform sensitivity in NGS-based MRD study. Herein, the sensitivity of $10^{-5}$ was verified by a standardized experimental design based on triplicate measurements of $1 \mu \mathrm{g}$ DNA input and 1 million sequencing reads using the LymphoTrack-MiSeq platform. MRD level was defined as the mean MRD burden of the triplicates. Two spike-in controls at concentrations of $0.001 \%$ tumor plasma cells (PC) for verifying the sensitivity of $10^{-5}$ and $0.01 \%$ (or $0.005 \%$ ) for MRD normalization were systematically analyzed. The spike-in control of $0.001 \%$ MRD was consistently detected in all samples, confirming a sensitivity of $10^{-5}$. Moreover, this standardized NGS approach yielded MRD measurements concordant with serological response and comparable to allele-specific oligonucleotide (ASO) real-time quantitative (RQ)-PCR. Moreover, NGS showed an improved sensitivity and provided quantification of MRD for cases assigned "positive but not quantifiable" (PNQ) by ASO RQ-PCR, even without the use of patient-specific probes/primers. Issues regarding the specificity of myeloma-specific sequences as MRD target, optimal input for spike-in normalization, and interpretation of MRD from triplicates are discussed. Herein, the standardized LymphoTrack-MiSeq-based method is verified to carry a sensitivity of $10^{-5}$, hence an effective tool for MRD monitoring in MM. As only a small number of samples are tested here, further study with a larger number of patients is warranted.

Keywords: minimal residual disease, multiple myeloma, next-generation sequencing, allele-specific oligonucleotide-PCR, sensitivity

\section{INTRODUCTION}

Correlation between depth of response and survival has long been established in multiple myeloma (MM) (1). Novel agent combinations have resulted in high rates of complete response (CR) (2). Despite this, a major portion of CR patients early or later on will eventually relapse, suggesting that low but clinically relevant levels of minimal residual disease (MRD) remain in the majority of patients attaining CR (3). Therefore, highly sensitive techniques are required to detect deeper response than clinical CR, as recently highlighted by the International Myeloma Working Group (IMWG) (4). 
Next-generation sequencing (NGS) of immunoglobulin (Ig) gene rearrangements has been applied to assess MRD and shown to be able to detect MRD at a level as low as $10^{-6}(5,6)$. Moreover, MRD negativity using the $10^{-5}$ threshold predicted for a superior progression-free survival (PFS) and overall survival (OS) of MM patients achieving at least very good partial response (VGPR), and improved PFS when CR patients are separately considered (7). Recently, the IMWG introduced the definition of MRDnegativity as the absence of clonal PC by either sequencingor flow cytometry-based techniques with a minimum sensitivity of $<10^{-5}$ (4) using the LymphoSIGHT (Sequenta/Adaptative) (7) and next-generation flow (NGF) EuroFlow approaches (8) as the reference methods, respectively. This is due to the fact that the majority of studies on the prognostic value of NGSbased MRD in MM were derived from a commercial service by LymphoSIGHT platform (Sequenta/Adaptive Inc.) $(7,9,10)$. More recently, MRD studies using this platform to evaluate the efficacy of daratumumab, a human IgGK anti-CD38 monoclonal antibody, have been reported $(11,12)$. Notably, the sensitivity was claimed to be of at least $10^{-5}(7,12), 10^{-6}(9,11)$, or $10^{-7}(10)$, depending on the different studies. In parallel, the LymphoTrack assay has become available as a commercial kit adapted to the detection of MRD by NGS and has been evaluated in MM (8, 13). However, these studies failed in providing an experimental validation of the sensitivity of $<10^{-5}$ for the LymphoTrack assay with variable (non-standardized) DNA inputs and depth of sequencing, which are essential for reproducible sensitivity among within and among distinct samples.

In this study we aimed to validate the sensitivity of $10^{-5}$ of a standardized workflow of LymphoTrack-MiSeq platform through detection of spike-in controls in follow-up MM samples and compare the NGS MRD results with those obtained by allelespecific oligonucleotide (ASO) real-time quantitative (RQ)-PCR.

\section{MATERIALS AND METHODS}

\section{Patients and Samples}

Four Chinese MM patients included in this study received autologous stem cell transplantation (ASCT) after VTD induction (bortezomib-thalidomide-dexamethasone) (14) or PAD (bortezomib-doxorubicin-dexamethasone) induction, followed by consolidation therapy using an additional two cycles of VTD or not, and then thalidomide maintenance (thalidomide $50 \mathrm{mg}$ daily) for 1 year. Diagnostic and follow-up bone marrow (BM) samples were studied. This study was approved by the Institutional Review Board of the University of Hong Kong/Hospital Authority Hong Kong West Cluster with informed

\footnotetext{
Abbreviations: NGS, next-generation sequencing; Ig, immunoglobulin; MRD, minimal residual disease; MM, multiple myeloma; PC, plasma cell; ASO, allelespecific oligonucleotide; RQ, real-time quantitative; PNQ, positive but not quantifiable; CR, complete response; PFS, progression-free survival; OS, overall survival; VGPR, very good partial response; NGF, next-generation flow; ASCT, autologous stem cell transplantation; BM, bone marrow; CDR3, complementaritydetermining region.
}

consents. Patient and sample characteristics are shown in Table 1.

\section{MRD Measurements by NGS}

Genomic DNA was extracted from BM samples using the QIAamp DNA Blood Mini Kit (Qiagen, Hilden, Germany). DNA concentration was measured by the Qubit dsDNA HS assay kit and Qubit 2.0 fluorometer (Thermo Fisher Scientific, Waltham, MA). DNA input was of $500 \mathrm{ng}$ for clonality assessment of the four diagnostic samples. PCR amplification of Ig gene rearrangement fragments was performed using the LymphoTrack IGH (FR1, FR2, FR3) and IGK assays according to the instructions of the manufacturer. Primers in the LymphoTrack assays were designed with Illumina adapters. Subsequently, each amplicon was purified by AMPure XP beads (Beckman Coulter, Brea, CA), followed by quantification using the KAPA Library Quantification Kit (Roche, Basel, Switzerland). An equal amount of all purified amplicons was pooled into a library of $4 \mathrm{nM}$, denatured, diluted, loaded on to the MiSeq, and subjected to MiSeq run (v3, $2 \times 300$ cycles; Illumina, San Diego, CA). Sequencing data in FASTQ format were analyzed using the LymphoTrack software package (InVivoScribe Technologies, San Diego, CA). A clonotype was defined when at least five identical sequencing reads were obtained. Frequency of a clonotype was determined by the number of sequencing reads of the clonotype divided by the total number of sequencing reads. A myeloma clonotype for tracking MRD was defined as an identical sequence with a frequency of $>5 \%$ as previously described (7).

For detection of MRD in the follow-up samples, triplicates of $1 \mu \mathrm{g}$ DNA input for each sample were subjected to PCR amplification using the LymphoTrack IGH (FR1) assay. DNA from a healthy donor BM was used as negative control. Subsequent library preparation and sequencing were performed as described above for clonality detection in diagnostic samples. The sequencing depth for each replicate was designed to be of 1 million sequencing reads. The number of cells contained in $1 \mu \mathrm{g}$ of each sample was validated by the real-time PCR standard curve method using plasmids, in which the albumin gene is cloned. Two plasmids containing unique IGH sequences were added to each replicate: one at the concentration of $10^{-5}$ (plasmid A: copy number equivalent to $0.001 \%$ of the number of total cells in a replicate) for validation of the sensitivity of $10^{-5}$, and the other at $5 \times$ $10^{-5}$ or $10^{-4}$ (plasmid B) for obtaining an amplification factor, i.e., percentage of tumor alleles per sequence read. The MRD level in each replicate was calculated from the corresponding reads of the myeloma-specific sequence and the amplification factor. The final MRD level of a sample was defined as the mean MRD levels of the triplicates. An overview of the MRD measurement method described above is shown in Figure 1.

\section{MRD Study by ASO RQ-PCR}

Clonality detection and subsequent MRD assessment by ASO RQ-PCR were performed as previously described (15). Clonality 
TABLE 1 | Patient and sample characteristics.

\begin{tabular}{|c|c|c|c|c|c|c|c|c|c|c|}
\hline \multirow{2}{*}{$\begin{array}{l}\text { Patient } \\
\text { ID }\end{array}$} & \multirow{2}{*}{ Isotype } & \multirow{2}{*}{$\begin{array}{l}\beta 2- \\
\text { Microglobulin, } \\
\mathrm{mg} / \mathrm{L}\end{array}$} & \multirow{2}{*}{$\begin{array}{l}\text { Albumin, } \\
\text { g/dL }\end{array}$} & \multirow{2}{*}{$\begin{array}{l}\text { LDH } \\
\text { high:1 }\end{array}$} & \multirow{2}{*}{$\begin{array}{l}\text { High-risk } \\
\text { cytogenetics* }\end{array}$} & \multirow{2}{*}{$\begin{array}{l}\text { Induction } \\
\text { regimen }\end{array}$} & \multirow{2}{*}{$\begin{array}{l}\text { Consolidation } \\
\text { therapy }\end{array}$} & \multicolumn{3}{|c|}{ Follow-up samples } \\
\hline & & & & & & & & ID & Time & $\begin{array}{l}\text { Clinical } \\
\text { response }\end{array}$ \\
\hline \multirow[t]{3}{*}{1} & $A$ & 3.0 & 3.9 & 0 & $\mathrm{t}(4 ; 14)$ & VTD & Yes & FU-1 & Before ASCT & VGPR \\
\hline & & & & & & & & FU-2 & After ASCT & $\mathrm{CR}$ \\
\hline & & & & & & & & FU-3 & After consolidation & $\mathrm{CR}$ \\
\hline \multirow[t]{2}{*}{2} & G & 9.1 & 3.3 & 0 & $\begin{array}{l}\text { t }(14 ; 16) \\
\text { del } 17 p\end{array}$ & VTD & Yes & FU-1 & $\begin{array}{l}\text { Before } \\
\text { consolidation }\end{array}$ & VGPR \\
\hline & & & & & & & & FU-2 & After consolidation & $\mathrm{CR}$ \\
\hline 3 & $\mathrm{D}$ & 13.6 & 4.2 & 0 & No & PAD & No & FU-1 & After ASCT & $\mathrm{CR}$ \\
\hline 4 & Non-secretory & 3.3 & 4.2 & 1 & No & VTD & No & FU-1 & After ASCT & $\mathrm{CR}$ \\
\hline
\end{tabular}

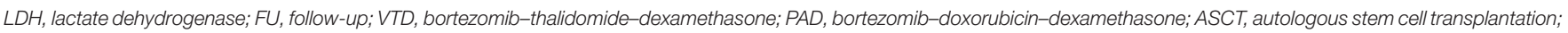
VGPR, very good partial response; $C R$, complete response.

*Presence of del(17p) and/or translocation $t(4 ; 14)$ and/or translocation $t(14 ; 16)$.

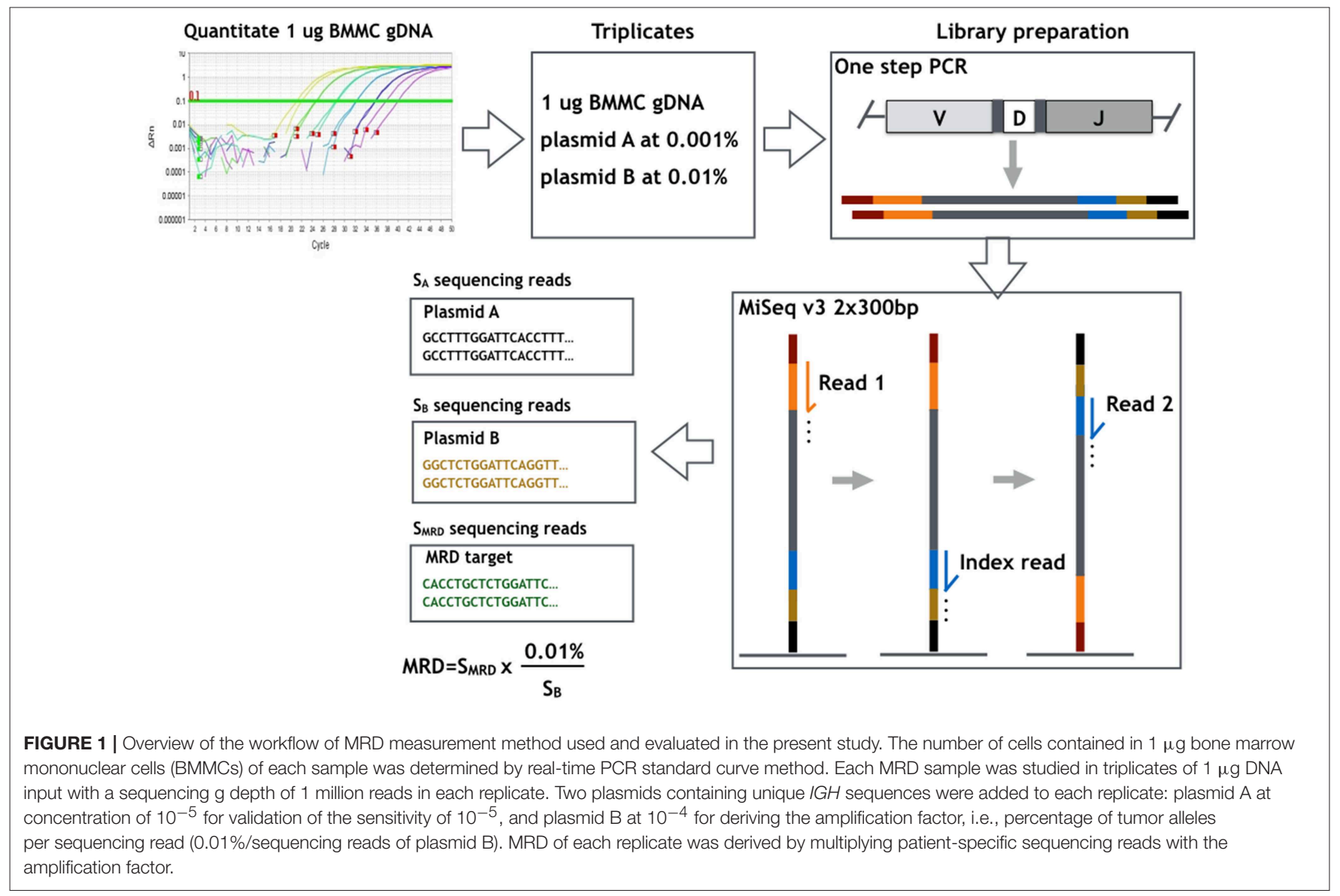

and the sequence of the complementarity-determining region 3 (CDR3) were identified by sequential PCR of the IGH VDJ, IGH DJ, and IGK VJ rearrangements, followed by Sanger sequencing. For MRD assessment, ASO forward primers with/without patient-specific reverse primers were designed. RQPCR was performed and interpreted according to the EuroMRD guidelines (16).

\section{RESULTS AND DISCUSSION}

\section{Assessment of Clonality in Diagnostic Samples}

Myeloma-specific clonal IGH VDJ rearrangements present in diagnostic MM BM samples were identified by IGH VDJ FR1 PCR in NGS. Patient 1 had two unrelated clonal rearrangements 
TABLE 2 | Results of clonality detection.

\begin{tabular}{|c|c|c|c|c|c|c|}
\hline \multirow[t]{2}{*}{ Patient ID } & \multirow[t]{2}{*}{ Clonality detected by Sanger sequencing } & \multicolumn{5}{|c|}{ Clonality detected by NGS } \\
\hline & & V-gene & D-gene & V-gene & Frequency & CDR3 identical to Sanger sequencing \\
\hline \multirow[t]{2}{*}{1} & Clone 1: $V_{H^{3}} 3.9(0)-1-7-(2) D 5.12(4)-2-(18) J_{H} 6$ & Clone 1 (IGHV3-9_01) & IGHD5-12_01 & IGHJ6_03 & 45.5 & $100 \%$ \\
\hline & Clone 2: $\mathrm{V}_{\mathrm{H}} 3.13(10)-5-(7) \mathrm{D} 3.22(10)-11-(1) \mathrm{J}_{\mathrm{H}} 3$ & Clone 2 (IGHV3-13_01) & IGHD3-22_01 & IGHJ3_02 & 18.5 & $100 \%$ \\
\hline 2 & $V_{H^{2}}$.5(2)-6-(2)D1.26(2)-3-(5) $J_{H} 4$ & IGHV2-5_09 & IGHD1-26_01 & IGHJ4_02 & 42.8 & $100 \%$ \\
\hline 3 & $V_{H} 3.66(0)-5-(15) D 3.16(7)-7-(14) J_{H} 6$ & IGHV3-66_02 & IGHD3-16_02 & IGHJ6_02 & 80.3 & $100 \%$ \\
\hline 4 & $V_{H} 3.21(1)-9-(8) D 2.21(3)-3-(5) J_{H} 6$ & IGHV3-21_02 & IGHD2-21_01 & IGHJ6_03 & 13.9 & $100 \%$ \\
\hline
\end{tabular}

NGS, next-gene sequencing; CDR3, complementarity-determining region.

(frequency of clonotype- 1 of $45.5 \%$ and of clonotype- 2 of $18.5 \%$ ), while patients $2-4$ had a single clonotype (frequency of $42.8,80.3$, and $13.9 \%$, respectively). The clonotype sequences identified by NGS were exactly the same as those derived from IGH multiplex consensus PCR followed by Sanger sequencing, including the two clonal rearrangements present in patient 1 . These two clonal rearrangements identified in patient 1 might occur in the same myeloma cell, or two independent myeloma cells, in case of biclonal disease. However, this could not be ascertained as one was a functional rearrangement, while the other was nonfunctional. This is consistent with the "allelic exclusion model of IgH locus rearrangements," whereby pro-B cells with a productive $\mathrm{V}(\mathrm{D}) \mathrm{J}$ rearrangement suppress $\mathrm{V}_{\mathrm{H}}$ to $\mathrm{DJ}_{\mathrm{H}}$ rearrangement of the second allele (17). Moreover, this model predicts that cells that make a non-functional $\mathrm{V}_{\mathrm{H}} \mathrm{DJ}_{\mathrm{H}}$ joint on the first allele will subsequently rearrange $\mathrm{V}_{\mathrm{H}}$ to $\mathrm{DJ}_{\mathrm{H}}$ on the second allele, resulting in the generation of mature lymphocytes carrying nonproductive $\mathrm{V}_{\mathrm{H}} \mathrm{DJ} \mathrm{J}_{\mathrm{H}}$ rearrangement on one allele, and productive rearrangement on the other (17). Results of clonality detection are shown in Table 2.

\section{NGS-Based MRD Detection Specificity of Sequences of Spike-In Control and MRD Target}

First of all, none of the $I G H$ sequences of the two plasmids added to each patient replicate sample were detected in the negative control, which used DNA from a healthy donor BM, indicating specificity of the sequences; hence, this indicates that, in principle, they were good candidates for spike-in controls. Subsequently, the feasibility of the clonal IGH VDJ rearrangements identified in the diagnostic samples as MRD targets in follow-up samples from the same patients was also evaluated. Clonal myeloma-specific sequences were not detected in the normal control except clonotype- 1 of patient 1 . The mean frequency of MRD from the triplicates of clonotype-1 identified in patient 1 were $1.5 \times 10^{-2}$ in sample follow-up-1, $7.7 \times 10^{-4}$ in follow-up-2, $4.3 \times 10^{-4}$ in follow-up-3, but only $2.2 \times 10^{-6}$ in the normal control, which is $2-4$ logs lower than the level of MRD detected in the MRD follow-up samples. While falsepositive MRD amplification may arise from non-specific primer binding to similar V-(D)-J sequences in normal lymphocytes, NGS MRD positivity is based on the detection of the identical myeloma clonal sequence (18). Therefore, MRD positivity in normal background in NGS might arise from normal cells producing the same sequence with myeloma cells (19). Indeed, identical IgH rearrangement with identical CDR3 sequences produced by unrelated cell populations has been observed in mice (20). However, there are 24 deleted/inserted nucleotides in the CDR3 region; hence, an identical clonotypic sequence generated by normal unrelated cells is unlikely (19). Another possibility is index misassignment when sequencing reads from pooled libraries are identified and sorted computationally by demultiplexing before data analysis $(21,22)$. On the other hand, DNA contamination is less likely. As patient 1 harbored two clonotypes including clonotype 2 , one would expect to find sequence of clonotype 2 , in addition to clonotype 1 , in the same normal control in case contamination had occurred. Moreover, while we are not able to analyze the number of tumor reads in the normal control due to the absence of a spike-in normalization plasmid, the number of reads would have ranged from 245 to 610 in sample FU-1 of patient 1 should there be one tumor cell in the FU sample. Given the small number of reads in the normal control, herein, $N=5,2$, and 0 in the respective replicate, we believe that index misassignment is the most likely reason.

\section{Sensitivity of NGS MRD Detection}

Regarding sensitivity, it should be noted that the $I G H$ sequence of $10^{-5}$ control plasmid was detected in at least one of the triplicates of all follow-up BM samples; thus, the sensitivity of $10^{-5}$ was achieved in all seven samples tested. Obtained sequencing reads for $10^{-5}$ control plasmid in each replicate are shown in Table 3. This is the first report in which the sensitivity of $10^{-5}$ was verified for the LymphoTrack-Miseq platform. Although the ability to detect MRD at the level of $10^{-6}$ disease burden has been demonstrated by serial dilutional experiments using the LymphoSIGHT platform $(5,6)$, a few points remain to be clarified in these experiments. First, MRD assessment was not the same for every follow-up sample, since the number of total input cells for MRD evaluation varied substantially (e.g., between 86,143 and $1,556,654$ cells) (5). Moreover, the ability to analyze a total of $10^{7}$ cells, which will entail analysis of between 5 and $50 \mu \mathrm{g}$ of DNA in one PCR reaction is technically challenging, if not currently impossible. Therefore, the exact technical details need to be provided to help clarify how the experiment was conducted. Finally, as the sensitivity of an MRD assay is dependent on the number of total input cells, a sensitivity of $10^{-6}$ might not be guaranteed in every sample. 
TABLE 3 | Results of minimal residual disease measured by next-generation sequencing and allele-specific oligonucleotide real-time quantitative-PCR.

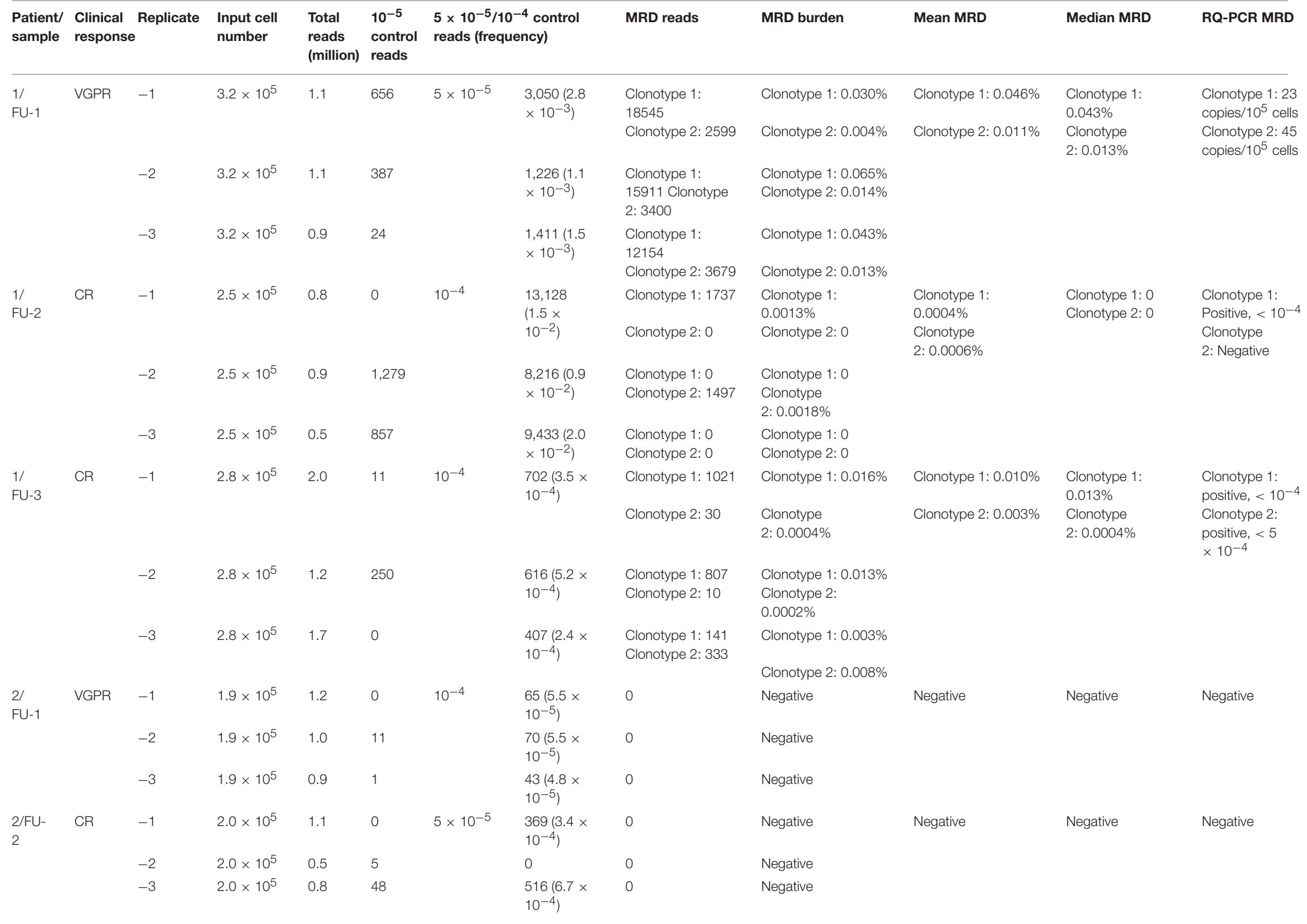




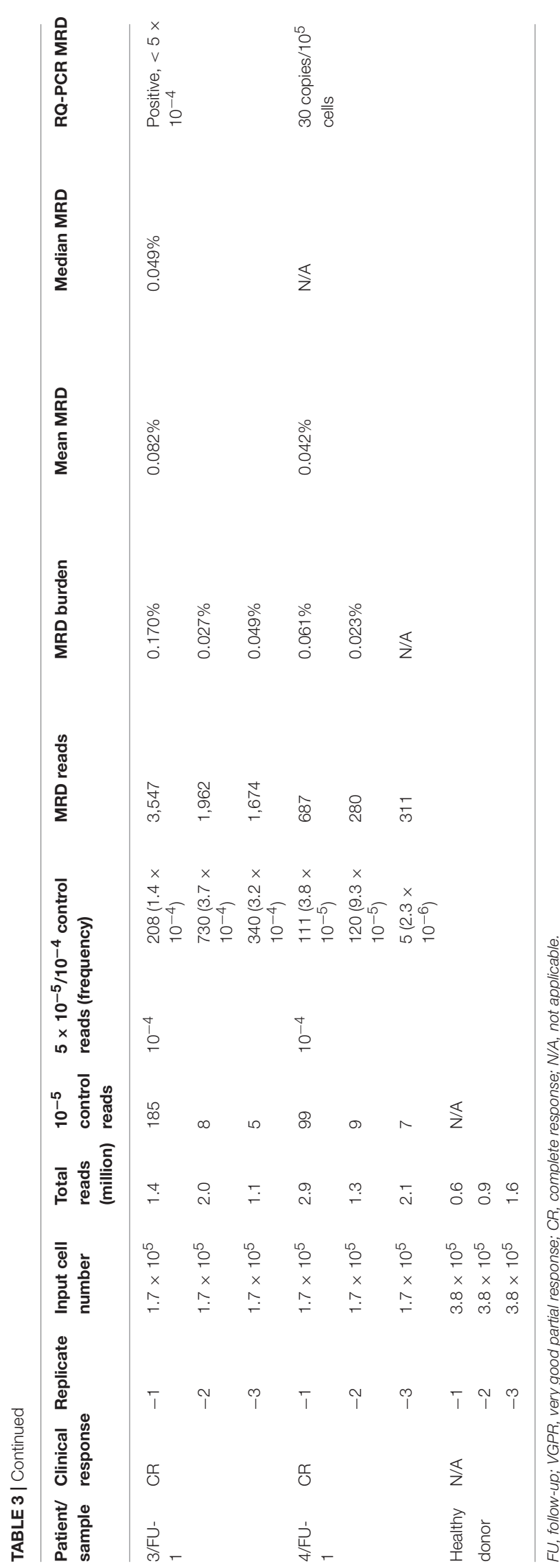

To analyze the prognostic impact or the efficacy of novel agents by MRD monitoring, some studies used pre-defined MRD cutoff levels (7, 10-12, 23), while others used MRDdetectable/undetectable as end-points $(13,24)$. In the latter reports, a uniform sensitivity for the MRD detection assay is important as MRD-negativity might refer to different levels of MRD in case sensitivity varies among distinct samples. Herein, we advocate each follow-up BM sample to be analyzed in triplicate with a standardized DNA input of $1 \mu \mathrm{g}$ per replicate for MRD detection to achieve a uniform sensitivity of $10^{-5}$, the minimal requirement to qualify for sequencing-based MRDnegativity according to the IMWG criteria (4). In turn, the Euro-MRD guidelines for ASO RQ-PCR recommend $500 \mathrm{ng}$ in triplicates for MRD assessment, whereby a sensitivity of $10^{-4}$ to $10^{-5}$ can be achieved. However, patient-specific primers/probes are often required with the ASO RQ-PCR approach, which is labor-intensive and time-consuming $(15,25)$.

\section{Molecular Number of Spike-In Control for Normalization}

Of note, with the approach here used, the MRD level was normalized by the amplification factor (percentage of tumor alleles per sequence read) obtained from the $5 \times 10^{-5}$ or $10^{-4}$ diluted plasmid. The LymphoSIGHT platform applied a pool of plasmids containing three unique IGH clonotypes to obtain a final amplification factor (number of molecules per sequence read) for absolute quantitation of tumor alleles (5). However, copy numbers of the plasmids have not been reported. A recent MRD study using the LymphoTrack-Miseq platform used spikein DNA corresponding to 1,000 clonal cells (13). Given the limited data in the field, the optimal copy number of spikein positive control DNA for absolute quantitation of tumor alleles still remains to be defined. Calculation of MRD levels using spike-in controls in NGS-based MRD assays is based on the same principles of using the standard curve in ASO RQ-PCR. According to the Euro-MRD guidelines for ASO RQ-PCR, dilution points included in the standard curve for quantitation of MRD in the follow-up samples should be within the "quantitative range," which requires the delta Ct of the three replicates to differ by $<1.5$, hence equivalent to a 2.8 -fold difference in copy number. At present, there is still no consensus for the spike-in control (the amplification factor) in NGSbased MRD assessment; however, it might be inferred that the concentration of this spike-in control should not be too low. In our study, concentrations of $5 \times 10^{-5}$ and $10^{-4}$, corresponding to approximately 10 and 20 copies of plasmids, were assessed. Sequencing reads (frequency) of $5 \times 10^{-5} / 10^{-4}$ plasmids are shown in Table 3. Indeed, our data showed that a wide variation in the frequency of plasmid reads was observed at the lower concentration of $5 \times 10^{-5}$ among the triplicates in one of two samples. Whether a high copy number (hundreds) of spike-in control may result in an inaccurate quantitation of very low MRD levels of tumor alleles needs to be confirmed. Nevertheless, a spike-in plasmid at approximately $10^{-4}$ appears appropriate for MRD normalization based on our limited experimental data, as variation lower than 2.8 -fold in frequency among triplicates of the $10^{-4}$ plasmid were achieved in four of five samples. 


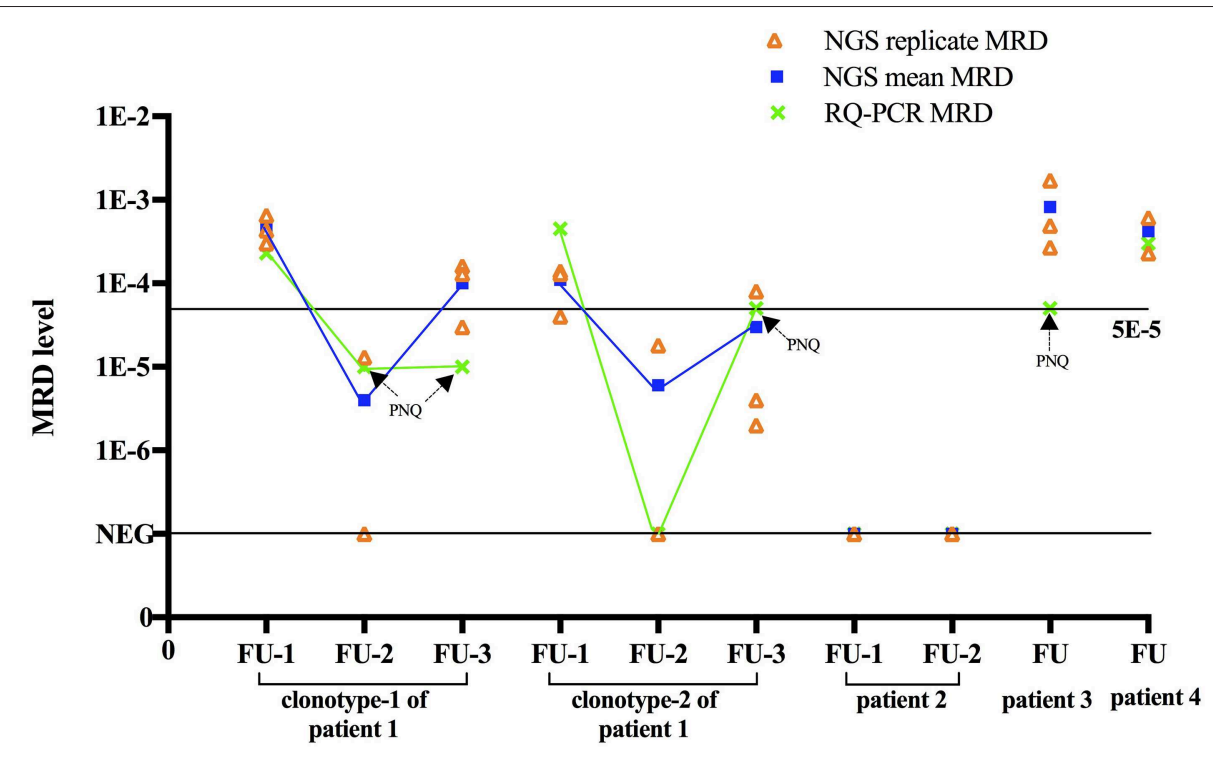

FIGURE 2 | Comparison between NGS and RQ-PCR. For each MRD sample, NGS based MRD of triplicates, mean MRD and ASO RQ-PCR based MRD are shown. For comparison purposes, cases that were defined as "positive not quantifiable" in ASO RQ-PCR were placed to sensitivity of that patient (10 ${ }^{-5}$ for clonotype-1 of patient $1,5 \times 10^{-5}$ for clonotype-2 of patient 1 and patients 3 ). Aberration: FU, follow-up; PNQ, positive not quantifiable.

However, in the follow-up sample of patient 4 , minor variation was observed in two of three replicates. Thus, MRD in this sample was determined by those two replicates and obtained comparable MRD level with ASO RQ PCR (42 copies vs. 30 copies, per $10^{5}$ cells).

\section{Interpretation of MRD From Triplicates}

Based on the approach here used, final MRD levels for a sample were derived from the mean MRD values of triplicates for that specific sample. In Euro-MRD guidelines for ASO RQPCR, an MRD result for a given follow-up sample might be interpreted as either quantifiable, positive but not quantifiable (PNQ), or negative (16). In NGS-based MRD assessment, MRD positivity is defined as the presence of two identical reads of the MRD target in the LymphoSIGHT platform and five identical reads in the LymphoTrack-Miseq approach. The percentage of MRD reads in a positive sample is calculated with the absolute myeloma molecules normalized by the spike-in control divided by total input cells in the reaction. Previously reported NGS MRD studies $(7,9-13,23,26)$ did not refer usage of MRD replicate measurements in follow-up samples; thus, the concept of PNQ for interpretation of MRD results has not yet been established in NGS-based MRD assays. Meanwhile, quantitative discrimination in NGS was regarded as super-imposable to its sensitivity, in contrast to ASO RQ-PCR, in which MRD levels beyond the quantifiable range are assigned "PNQ" (26). Indeed, a certain MRD level can be reached in MRD samples as long as tumor-specific alleles are detected by NGS. Moreover, an improved quantitative ability (greater sensitivity) is observed in NGS compared to RQ-PCR. For instance, Faham et al. reported that LymphoSIGHT was highly quantitative for frequencies above $10^{-5}(5)$, while frequencies of $10^{-4}$ to $10^{-5}$ were usually detectable but not quantifiable in ASO RQ-PCR. Here, a higher quantifiable range of NGS compared to ASO RQ-PCR was observed for the first follow-up sample of patient 1. For MRD assessment by ASO RQ-PCR, ASO forward primers and patientspecific reverse primers were designed for clonotypes 1 and 2, achieving a quantitative range of $10^{-4}$ and $5 \times 10^{-4}$, respectively. If similar criteria of quantitative range in ASO RQ-PCR (i.e., delta $\mathrm{Ct}$ of the three replicates to differ by no more than 1.5) are applied to NGS (i.e., 2.8-fold difference in frequency in triplicates), the spike-in control of $5 \times 10^{-5}$ in this same could be regarded as quantifiable. However, MRD measured from a single replicate by NGS is not necessarily accurate. In fact, random errors increased at clonotype frequencies below $10^{-5}$ in the serial dilutional experiments performed by Faham et al. (5). Similarly, triplicates applied in our study also clearly showed that despite residual disease was detected at very low MRD levels of $10^{-5}$, they were not reproducible among replicates, consistent with Poisson statistics for low numbers of target molecules, a limitation that would potentially be overcome by increasing the DNA input. Therefore, we applied triplicates of $1 \mu \mathrm{g}$ DNA for MRD measurement for each follow-up sample and MRD level was defined as mean MRD burden of the triplicates. Results of measured MRD are shown in Table 3 and Figure 2.

\section{Comparison Between NGS and ASO-PCR to Evaluate MRD}

In the four patients here studied by NGS, MRD was also analyzed in parallel by ASO RQ-PCR using patient-specific primers, rendering a sensitivity of $5 \times 10^{-5}$ to $10^{-5}$. As a result, MRD was detected by ASO RQ-PCR in the five follow-up samples of patients 1,3 , and 4 but not in the two follow-up samples of patient 
2. Notably, NGS yielded MRD+ or MRD- results concordant with ASO RQ-PCR in all these seven follow-up samples.

The dynamic change of MRD in the three sequential MRDpositive samples obtained by NGS and ASO-PCR was fully concordant. The serological response of patient 1 was VGPR for the follow-up-1 sample and CR for the follow-up-2 and follow-up-3 samples. Correspondingly, MRD of clonotype 1 was reduced from $0.045 \%$ in sample follow-up- 1 to PNQ (positive but non-quantifiable as MRD level is below the quantitative range, $10^{-4}$, of this patient) in samples at follow-up- 2 and -3 by ASO RQ-PCR, and reduced from 0.046 to $0.0004 \%$ and $0.010 \%$ by NGS (Figure 2). Therefore, both methods showed the expected decrease of MRD levels associated with the change in serological response. In addition, clonotype 2 of patient 1 was detected in the follow-up-2 sample by NGS but not by ASO RQ-PCR. This would be attributed to the higher sensitivity of NGS $\left(10^{-5}\right)$ compared to ASO RQ-PCR $\left(5 \times 10^{-5}\right)$ for clonotype 2. Ladetto et al. compared MRD in sequential follow-up samples in MM by NGS and ASO RQ-PCR, and discordances were observed in $44 \%(20 / 45)$ of the samples (26). Among the 20 discordant cases, ASO RQ-PCR yielded a positive result in 8 that were negative by NGS MRD, indicating that this discordance could not be accounted for by the different sensitivity between the two approaches. Therefore, further studies are needed to understand the reasons for the discordant MRD results obtained with NGS and ASO RQ-PCR, as discordant MRD results post-treatment by these two techniques would generate different conclusions about the MRD response.

In summary, this report provides the first data on a standardized protocol for MRD assessment using the LymphoTrack-Miseq platform based on the use of triplicates of $1 \mu \mathrm{g}$ DNA input for each MRD sample and a sequencing depth of 1 million sequencing reads per sample. In addition, in the proposed protocol, two spike-in controls were also included:

\section{REFERENCES}

1. Kapoor P, Kumar SK, Dispenzieri A, Lacy MQ, Buadi F, Dingli D, et al. Importance of achieving stringent complete response after autologous stem-cell transplantation in multiple myeloma. J Clin Oncol. (2013) 31:4529-35. doi: 10.1200/jco.2013.4 9.0086

2. Mailankody S, Korde N, Lesokhin AM, Lendvai N, Hassoun H, StetlerStevenson $\mathrm{M}$, et al. Minimal residual disease in multiple myeloma: bringing the bench to the bedside. Nat Rev Clin Oncol. (2015) 12:286-95. doi: 10.1038/nrclinonc.2014.239

3. Paiva B, van Dongen JJ, Orfao A. New criteria for response assessment: role of minimal residual disease in multiple myeloma. Blood. (2015) 125:3059-68. doi: 10.1182/blood-2014-11-568907

4. Kumar S, Paiva B, Anderson KC, Durie B, Landgren O, Moreau P, et al. International myeloma Working Group consensus criteria for response and minimal residual disease assessment in multiple myeloma. Lancet Oncol. (2016) 17:e328-e46. doi: 10.1016/s1470-2045(16)30206-6

5. Faham M, Zheng J, Moorhead M, Carlton VE, Stow P, CoustanSmith E, et al. Deep-sequencing approach for minimal residual disease detection in acute lymphoblastic leukemia. Blood. (2012) 120:5173-80. doi: 10.1182/blood-2012-07-444042

6. Logan AC, Zhang B, Narasimhan B, Carlton V, Zheng J, Moorhead M, et al. Minimal residual disease quantification using consensus primers and one serving as a $10^{-5}$ sensitivity marker and the other as a reference for MRD normalization. Moreover, the standardized NGS protocol proposed yielded MRD results comparable to ASO RQ-PCR, both of which were concordant with the serological response. Furthermore, NGS proved to achieve an improved sensitivity and more reproducible quantification of low levels of MRD, otherwise declared PNQ by ASO RQ-PCR, without the need for patient-specific probe/primers and, hence, a less labor-intensive technique and faster turn-around time. As only a small number of samples are tested here, further study with a larger number of patients is warranted.

\section{ETHICS STATEMENT}

This study was carried out in accordance with the recommendations of the Institutional Review Board of the University of Hong Kong/Hospital Authority Hong Kong West Cluster with informed consents. All subjects gave written informed consent in accordance with the Declaration of Helsinki. The protocol was approved by the Institutional Review Board of the University of Hong Kong/Hospital Authority Hong Kong West Cluster.

\section{AUTHOR CONTRIBUTIONS}

CC designed and supervised the study. QY and YB performed the experiments. All authors analyzed the data, wrote the manuscript, and approved the final version.

\section{FUNDING}

This work was supported by Hong Kong Blood Cancer Foundation and National Natural Science Foundation of China (81470369) awarded to CC. high-throughput IGH sequencing predicts post-transplant relapse in chronic lymphocytic leukemia. Leukemia. (2013) 27:1659-65. doi: 10.1038/leu.2013.52

7. Martinez-Lopez J, Lahuerta JJ, Pepin F, Gonzalez M, Barrio S, Ayala $\mathrm{R}$, et al. Prognostic value of deep sequencing method for minimal residual disease detection in multiple myeloma. Blood. (2014) 123:3073-9. doi: 10.1182/blood-2014-01-550020

8. Flores-Montero J, Sanoja-Flores L, Paiva B, Puig N, Garcia-Sanchez O, Bottcher S, et al. Next generation flow for highly sensitive and standardized detection of minimal residual disease in multiple myeloma. Leukemia. (2017) 31:2094-103. doi: 10.1038/leu.2017.29

9. Avet-Loiseau H, Lauwers-Cances V, Corre J, Moreau P, Attal M, Munshi N. Minimal residual disease in multiple myeloma: final analysis of the IFM2009 trial. Blood. (2017) 130 (Suppl 1):435.

10. Takamatsu H, Takezako N, Zheng J, Moorhead M, Carlton VEH, Kong KA, et al. Prognostic value of sequencing-based minimal residual disease detection in patients with multiple myeloma who underwent autologous stem-cell transplantation. Ann Oncol. (2017) 28:2503-10. doi: 10.1093/annonc/mdx340

11. Dimopoulos MA, White DJ, Benboubker L, Cook G, Leiba M, Morton J, et al. Daratumumab, lenalidomide, and dexamethasone (DRd) versus lenalidomide and dexamethasone $(\mathrm{Rd})$ in relapsed or refractory multiple myeloma (RRMM): updated efficacy and safety analysis of pollux. Blood. (2017) 130(Suppl 1):739.

12. Mateos MV, Dimopoulos MA, Cavo M, Suzuki K, Jakubowiak AJ, Knop S, et al. Phase 3 randomized study of daratumumab plus bortezomib, melphalan, and 
prednisone (D-VMP) versus bortezomib, melphalan, and prednisone (VMP) in newly diagnosed multiple myeloma (NDMM) patients (Pts) ineligible for transplant (ALCYONE). Blood. (2017) 130(Suppl 1):LBA-4-LBA.

13. Medina A, Jiménez C, Puig N, Sanchez-Vega B, Flores-Montero J, González $\mathrm{M}$, et al. New alternatives for the evaluation of minimal residual disease (MRD) detection by next generation sequencing in multiple myeloma. Blood. (2017) 130 (Suppl 1):1783.

14. Chim CS, Lie AK, Chan EY, Liu HS, Lau CW, Yip SF, et al. Treatment outcome and prognostic factor analysis in transplant-eligible Chinese myeloma patients receiving bortezomib-based induction regimens including the staged approach, PAD or VTD. J Hematol Oncol. (2012) 5:28. doi: 10.1186/1756-8722-5-28

15. Bai Y, Wong KY, Fung TK, Chim CS. High applicability of ASORQPCR for detection of minimal residual disease in multiple myeloma by entirely patient-specific primers/probes. J Hematol Oncol. (2016) 9:107. doi: 10.1186/s13045-016-0336-4

16. van der Velden VH, Cazzaniga G, Schrauder A, Hancock J, Bader P, Panzer-Grumayer ER, et al. Analysis of minimal residual disease by Ig/TCR gene rearrangements: guidelines for interpretation of real-time quantitative PCR data. Leukemia. (2007) 21:604-11. doi: 10.1038/sj.leu.24 04586

17. Jung D, Giallourakis C, Mostoslavsky R, Alt FW. Mechanism and control of $\mathrm{V}(\mathrm{D}) \mathrm{J}$ recombination at the immunoglobulin heavy chain locus. Ann Rev Immunol. (2006) 24:541-70. doi: 10.1146/annurev.immunol.23.021704.1 15830

18. Kotrova $M$, van der Velden VHJ, van Dongen JJM, Formankova R, Sedlacek P, Bruggemann M, et al. Next-generation sequencing indicates false-positive MRD results and better predicts prognosis after SCT in patients with childhood ALL. Bone Marrow Transplant. (2017) 52:962-8. doi: 10.1038/bmt.2017.16

19. Moppett J, Wakeman S, Duez M, Bartram J, Wright G, Hubank $\mathrm{M}$, et al. Immunoglobulin/T-cell receptor (Ig/TCR) allele usage in normal and on treatment bone marrow samples in childhood acute lymphoblastic leukaemia-implications for NGS based MRD analysis. Blood. (2016) 128:4073.
20. Seidl KJ, MacKenzie JD, Wang D, Kantor AB, Kabat EA, Herzenberg LA, et al. Frequent occurrence of identical heavy and light chain Ig rearrangements. Int Immunol. (1997) 9:689-702.

21. Li Q, Zhao X, Zhang W, Wang L, Wang J, Xu D, et al. Reliable multiplex sequencing with rare index mis-assignment on DNB-based NGS platform. BMC Genom. (2019) 20:215. doi: 10.1186/s12864-019-5569-5.

22. Effects of Index Misassignment on Multiplexing and Downstream Analysis (white paper). Available online at: https://www.illumina.com/content/dam/ illumina-marketing/documents/products/whitepapers/index-hoppingwhite-paper-770-2017-004.pdf

23. Martinez-Lopez J, Sanchez-Vega B, Barrio S, Cuenca I, Ruiz-Heredia Y, Alonso R, et al. Analytical and clinical validation of a novel in-house deepsequencing method for minimal residual disease monitoring in a phase II trial for multiple myeloma. Leukemia. (2017) 31:1446-9. doi: 10.1038/leu.2017.58

24. Cheng S, Inghirami G, Cheng S, Tam W. Simple deep sequencing-based postremission MRD surveillance predicts clinical relapse in B-ALL. J Hematol Oncol. (2018) 11:105. doi: 10.1186/s13045-018-0652-y

25. Bai Y, Orfao A, Chim CS. Molecular detection of minimal residual disease in multiple myeloma. Br J Haematol. (2018) 181:11-26. doi: 10.1111/bjh.15075

26. Ladetto M, Bruggemann M, Monitillo L, Ferrero S, Pepin F, Drandi D, et al. Next-generation sequencing and real-time quantitative PCR for minimal residual disease detection in B-cell disorders. Leukemia. (2014) 28:1299-307. doi: $10.1038 /$ leu. 2013.375

Conflict of Interest Statement: The authors declare that the research was conducted in the absence of any commercial or financial relationships that could be construed as a potential conflict of interest.

Copyright (c) 2019 Yao, Bai, Orfao and Chim. This is an open-access article distributed under the terms of the Creative Commons Attribution License (CC BY). The use, distribution or reproduction in other forums is permitted, provided the original author(s) and the copyright owner(s) are credited and that the original publication in this journal is cited, in accordance with accepted academic practice. No use, distribution or reproduction is permitted which does not comply with these terms. 